\title{
CASCOS UTILIZADOS POR LOS PUEBLOS DE LAS ESTEPAS EUROASIÁTICAS EN LA ÉPOCA DE LOS ESCITAS Y DE LOS SÁRMATAS
}

\author{
POR
}

\author{
B. BRENTJES
}

\section{RESUMEN - ABSTRACT}

El casco apareció en las estepas de Eurasia hacia el final del II milenio a.C. Los cascos eran forjados y parecen derivar de los cascos chinos del periodo Shang-Yin. Este tipo de casco alcanzó Europa oriental durante los primeros siglos del primer milenio a.C. Un nuevo desarrollo fue el capacete blindado de fieltro con escamas de hierro. Fue creado por los escitas bajo la influencia de las corazas asirias de escamas que encontraron durante sus incursiones en el Próximo Oriente. Tres cascos asirios apuntados han sido hallados en el norte del Cáucaso y podrían ser botín o adquisiciones de los Cimerios o los escitas durante los siglos VII-VI a.C.

Durante los siglos V-IV a.C. los griegos vendieron diversos tipos de cascos a los Escitas. Cascos tracios pueden haber sido capturados durante campañas en los Balcanes. Cascos Célticos hallados en diversos yacimientos pudieron importarse via Italia -o haber pertenecido a mercenarios celtas de los reyes del Ponto.

The helmet appeared in the steppes of Eurasia at the end of the second mill. BC. The helmets were cast and seem to follow Chinese helmets of the Shang-Yin time. This type of helmet reached eastern Europe in the first centuries of the first mill. BC. A new development was the armoured cap of felt with iron casted scales.It was developed by the Skythians under the impression of the Assyrian scale armour which they met during their invasion in Near East. Three Assyrian pointed helmets were found in northern Caucasia and might be booty or bought by the Cimmerians or the Skythians in the Seventh or sixth century BC.

During the fifth and fourth centuries the Greeks sold several types of helmets to the Skythians. Thrakian helmets might have been won during wars on the Balkans. Celtic helmets found at several sites might have been imported via Italy -or are left by Celtic mercenaries of the Pontos kings.

\section{PALABRAS CLAVE - KEY WORDS}

Sistemas de defensa de los guerreros nómadas. Cascos del Kuban. Prototipos chinos. Capacetes con protección de escamas. Tecnología asiria. Importación de cascos asirios. Cascos griegos de las estepas. Importaciones tracias y griegas.

Defence systems of nomadic warriors. Kuban helmets. Chinese prototypesScale armour at caps. Assyrian technology. Import of Assyrian helmets. Greek helmets in the steppes. Thrakian and Celtic imports.

Desde el primer milenio a. de C. la dotación de armas de los nómadas incluía diversos elementos de protección para el propio cuerpo y para la cabalgadura. Los elementos de protección más importantes utilizados por los escitas, según la impresión de los griegos, aparecen, entre otros, en la cimera de Solocha: casco, armadura y dos tipos de escudo. Son reproducciones auténticas de armas escitas, si bien el casco que reproducimos aquí parece ser una importación de Grecia. Las armas defensivas y ofensivas representadas en la cimera de Solo- 
cha están totalmente comprobadas desde el punto de vista arqueológico y, junto a ellas, también otras formas. Sin embargo esto no debe hacernos pasar por alto los problemas que surgen del hecho de que la mayoría de las armas defensivas no estaban hechas de metal sino de materiales perecederos como madera, cuero y tejidos con lo que se han perdido casi todas ellas. Además, en la comparación de los legados de los (euro)escitas y sármatas con los de los saces y los de otros orígenes de las regiones al Este del Ural se ponen de manifiesto dos factores histórico-culturales con diferencias regionales y temporales.

Los escitas - y a continuación de ellos los sármatas- vivieron dentro de campo de visión de los griegos y después de los romanos, muy aficionados a las ilustraciones, que nos legaron reproducciones relativamente exactas de sus aliados o de sus adversarios en el ámbito de las estepas. De la región oriental de las estepas, hasta entrada la alta Edad Media, existen fundamentalmente sólo los relieves, bastante uniformes, con las delegaciones de los vasallos de los aqueménidas de Persépolis y algunas respresentaciones propias. Las primeras fuentes gráficas ilustrativas son las pinturas murales sogdianas del siglo VI-VIII. Desgraciadamente la mayoría de ellas son de carácter religioso y épico y por lo tanto deben interpretarse con cautela.

Las distintas costumbres funerarias influyeron de modo muy terminante así como el comportamiento respecto a las sepulturas existentes. Al Oeste del Ural predominaba el entiero del cuerpo acompañado de ofrendas y evidentemente existía un temor relativamente efectivo a la tumba, con lo que se mantenían a raya los saqueos. En las estepas orientales y en los oasis fluviales se imponía, aunque con límites regionales y temporales, la exposición del cadáver, seguida de un enterramiento parcial sin osarios, pero junto a ello existía la incineración, en la que a menudo sólo se conservaba una parte de las ofrendas. Allí, el saqueo, especialmente de las tumbas ricas, poco después del entierro, era más frecuente que en las estepas europeas. A ello se añadió que, durante los siglos XVI y XVII, los ladrones de tumbas rusos abrieron casi todos los kurganos (túmulos) y se llevaron todo aquello en lo que no habían reparado sus predecesores. La consecuencia es que conocemos muchas sepulturas lujosas, a menudo intactas, situadas en la región al Oeste del Volga mientras que en el Este existe una ausencia casi total de hallazgos semejantes. Los ladrones de tumbas se llevaron primordialmente los objetos de metales preciosos y las armas. Sólo en casos excepcionales se conservaron los textiles, como por ejemplo en las "lentes de hielo" naturalesen los kurganos de Pazyryk.

Además deben tenerse en cuenta las relaciones que mantuvieron durante siglos los escitas y los sármatas con las ciudades del Mar Negro y los estados balcánicos. En cierta medida, llevaron armas defensivas antiguas al territorio de las estepas, mientras que en el Este hay que hablar más bien de una transferencia de elementos de combate de los nómadas en el Irán.

Queda por examinar, en particular, el papel desempeñado por la influencia china, entendiéndose aquí como "chinas" las múltiples agrupaciones étnicas y culturales en el territorio al Este del Altai hasta el Pacífico, que aún no han sido bien analizadas entre el segundo milenio y los principios del primer milenio a. de C. Parecen existir grandes lagunas en la investigación de los territorios que habitan ahora sobre todo los mongoles y en la de los desiertos limítrofes al Sur.

Tomando como base la problemática que hemos expuesto, en las secciones siguientes se describirá en primer lugar la situación actual de los hallazgos -incluidas las representaciones artísticas- y después se intentará realizar una interpretación histórica.

E.V. Černenko ${ }^{1}$, distinguido por su Historia de las Armas de las Estepas, indicó en 1968 una cifra de 62 hallazgos de cascos, parte de los cuales ha quedado hasta ahora sin publicar y algunas piezas se han perdido. Entretanto, se han añadido además nuevos hallazgos. Según sus indicaciones, la mitad de los cascos procede de hallazgos en tumbas. Un yacimiento en

\footnotetext{
1 Černenko, 1968.
} 
Olonest en Moldavia contenía seis cascos, parte del tipo ático, y parte del tipo "tracio". Aquí se tropieza con el problema de la clasificación de cada uno de los hallazgos, que es parcialmente contradictoria en la bibliografía, sobre todo en lo que se refiere al casco "tracio", una definición que se remonta a Schröder ${ }^{2}$. Se basa en la relativa frecuencia de los hallazgos de cascos no griegos o no romanos en el Este de la Península de los Balcanes, que se atribuyen a los tracios o a los getas. Bajo la influencia del supuesto de un centrismo grecorromano, se les considera con frecuencia como formas antiguas "barbarizadas". El propio Cernenko sostiene la opinión de que los cascos de metal del territorio de las estepas se remontaban exclusivamente a fuentes "occidentales", incluyendo en ello hasta los llamados cascos "Kuban", un grupo de cascos de bronce encontrados en su mayoríaen el territorio de Kuban. Es cierto que consideró que esta forma de casco aparece también en el Este - en el Altai- y acredita un casco "antiguo" del territorio de Astrajan ${ }^{3}$, pero pasó por alto los cascos de metal de China que eran más antiguos. Por el contrario, W.V. Gorelik ${ }^{4}$ pone de relieve las relaciones respecto a técnica de armamento entre los pueblos de las estepas y China, aunque no tiene en cuenta los hallazgos de Anyang de los siglos XIV-XII a. de C. ${ }^{5}$ Cernenko ordena los hallazgos en los grupos siguientes:

1. Importaciones de cascos áticos, corintios, calcídicos y "tracios" (Fig. I) del ámbito griego del siglo $\mathrm{V}$ a. de $\mathrm{C}$. Incluye aquí también el grupo más antiguo de los cascos "Kuban". Cita seis ejemplares, cinco de los cuales pertenecen todavía al siglo VI a. de C. , los cascos de Kelermes, Krymskaja, Peceur en el territorio sármata del Volga, Samarkanda y Kysmyci en Kirguisia. El casco de Krymskaja presenta restos de una decoración de oro que han sido considerados como parte de una corona. Todavía más antiguo podría ser el llamado frecuentemente casco "griego" encontrado en la Staniza Machoserskaja. En Romejskovka, Elisavetinskaja, Kertsch y Phangoria se han descubierto cascos corintios. Una estatua de Kertsch y una figura de jinete de la cimera de Solocha llevan también cascos de este tipo. Un casco calcídico proviene de la tumba 16 de Nymphaion ${ }^{6}$.

2. Cernenko cita diecisiete cascos áticos en los siglos V-III a. de C., tales como los de Temnolesskaja, Pchie, Pastyrskaja ${ }^{7}$, Kekuvatskaja, Volkovcy y otros lugares. En Olonest ${ }^{8}$ y Mastjugino se encontraron "cascos tracios" - Cernenko propuso para este tipo de casco en forma de gorro "frigio" la denominación de casco "frigio-ático". Un segundo tipo con "cresta" y carrilleras? se adscribe igualmente al ámbito tracio. Está presente en Bubuja yen Kertsch (Fig. VI-VII).

3. Černenko considera como cascos de adscripción antigua los cascos forjados del tipo "Kuban" - el grupo más antiguo es de fundición. Junto a ellos se llevaban también cascos áticos -Olbia—, corintios y "tracios". En este último grupo indica el casco de Nimphaion, hallado en 1868 y conservado actualmente en Oxford. Sólo de Dalmacia, Cernen$\mathrm{ko}^{10}$ cita treinta ejemplares. Pero esto tropieza una vez más con el problema de poner en el mismo plano la manufactura de los hallazgos y el territorio de procedencia. Esta tesis de gran aceptación puede ser cierta pero también puede ser equivocada. El acompañamiento con ricos dones funerarios depende de los supuestos religiosos pero no de la certeza de

2 Schröder, 1912.

3 Helm von Nikolskoe. Černenko, 1968, Abb. 40.

4 Gorelik, 1987, S. 110-133.

5 Müller-Karpe, 1980, Taf. 583 und 587.

6 Černenko, 1968, Abb. 45, 1.

El casco Pastyrskaja aparece en la bibliografía también con el nombre de Galuscino, que es como se llama el bosque de Pastyrskaja en el que se halló el casco. En algunos autores aparecen ambos nombres uno junto a otro.

8 Černenko, 1968, Abb. 45,3 und 4.

9 Černenko, 1968, Abb. 47.

10 Černenko, 1968, Abb. 51. 
ser los creadores de dichos objetos. Naturalmente la ofrenda presupone que se tiene a disposición el objeto, pero el análisis de los grupos culturales que ponen ricos dones en las tumbas, clasifica a dichos grupos en un orden social relativamente antiguo, en el que se imponían como norma las ofrendas de prestigio (Fig. V).

4. La forma del casco de Nymphaion, una semiesfera adornada con dos "crestas" y con visera, lo relaciona con formas orientales primitivas. También merece una discusión más detallada el casco de la Staniza Dachovskaja en el territorio de Kuban ${ }^{11}$ (Fig. XI, 3), que presenta elementos - cuernos y ojos - de una cabeza de carnero. Cernenko menciona un casco, que se ha perdido, que llevaba como adorno una cabeza de caballo estilizada. También se pueden citar cascos, con figuras de animales, de los celtas, los tracios, los partos y de Asia Central. Los ojos muy marcados del casco de Dachover recuerdan el adorno de los ojos de los lujosos cascos tracios ${ }^{12}$ así como a las máscaras Ta'o-t’ieh de los cascos chinos.

Černenko ya menciona una forma específica de las estepas, la capucha de cuero ${ }^{13}$ reforzada con láminas protectoras, pero sin poderla reconstruir con el material del que dispone (Fig. VIII). Esta situación ha cambiado con el hallazgo de una capucha-casco de esta forma en el Ingul ${ }^{14}$,de modo que este tipo de casco pertenece comprobadamente al tiempo de los escitas. Las láminas protectoras son de hierro, tal como lo demuestran los hallazgos de la tumba 401 de Zurovch, Cistopol, de la Kamennaja Mogila y del kurgan 4 de Vernieevka en Poltowa. Al parecer, se ha perdido ${ }^{15}$ un casco de esta clase procedente del Vasjurinaja Gora y citado por M. Rostovzeff.

Las comprobaciones siguientes se basan en la bibliografía accesible y no pueden ser completas.

Los cascos originales existentes en la actualidad, o sus fragmentos, son principalmente de bronce. Leskov ${ }^{16}$ indica el latón como material de dos cascos del kurgan 3 del lago Cetuk, una indicación que probablemente se puede aceptar a la vista de la alta proporción de cinc de las puntas de flecha escitas. Se trata de cascos-capucha planos que se ponen sobre un forro de cuero. Pertenecen, como forma tardía, al grupo de cascos que se denominade tipo "Kuban”. Galanina ${ }^{17}$ reúne bajo este término los hallazgos siguientes (Fig. I):

\author{
1, 2 y 3 Kelermes \\ 4 Krymskaja, kurgano Voroncovskij \\ 5 Krymskaja, kurgano Mezepinskij \\ 6 Gvaderskoe \\ 7 Staryj Pečeur \\ 8 y 9 sin indicación de lugar \\ 10 Siberia occidental \\ 11 Samarkanda \\ 12 Kysmyci \\ (falta el casco del Altai) $^{18}$
}

\footnotetext{
11 Černenko, 1968, Abb. 53, 3.

12 Berciu, 1974, Fig. 3-7 u.a.

13 Černenko, 1968, S. 97 f.

14 Šapošnikova, 1970, S. 208-212, Abb. 4.

15 Černenko, 1968, S. 97 f.

16 Leskov, Katalognr. 242 und 243, Abb. 198 und 199.

17 Galanina, 1985, S. 169-183.

18 Gorelik, 1987. Abb. 6, 1.
} 


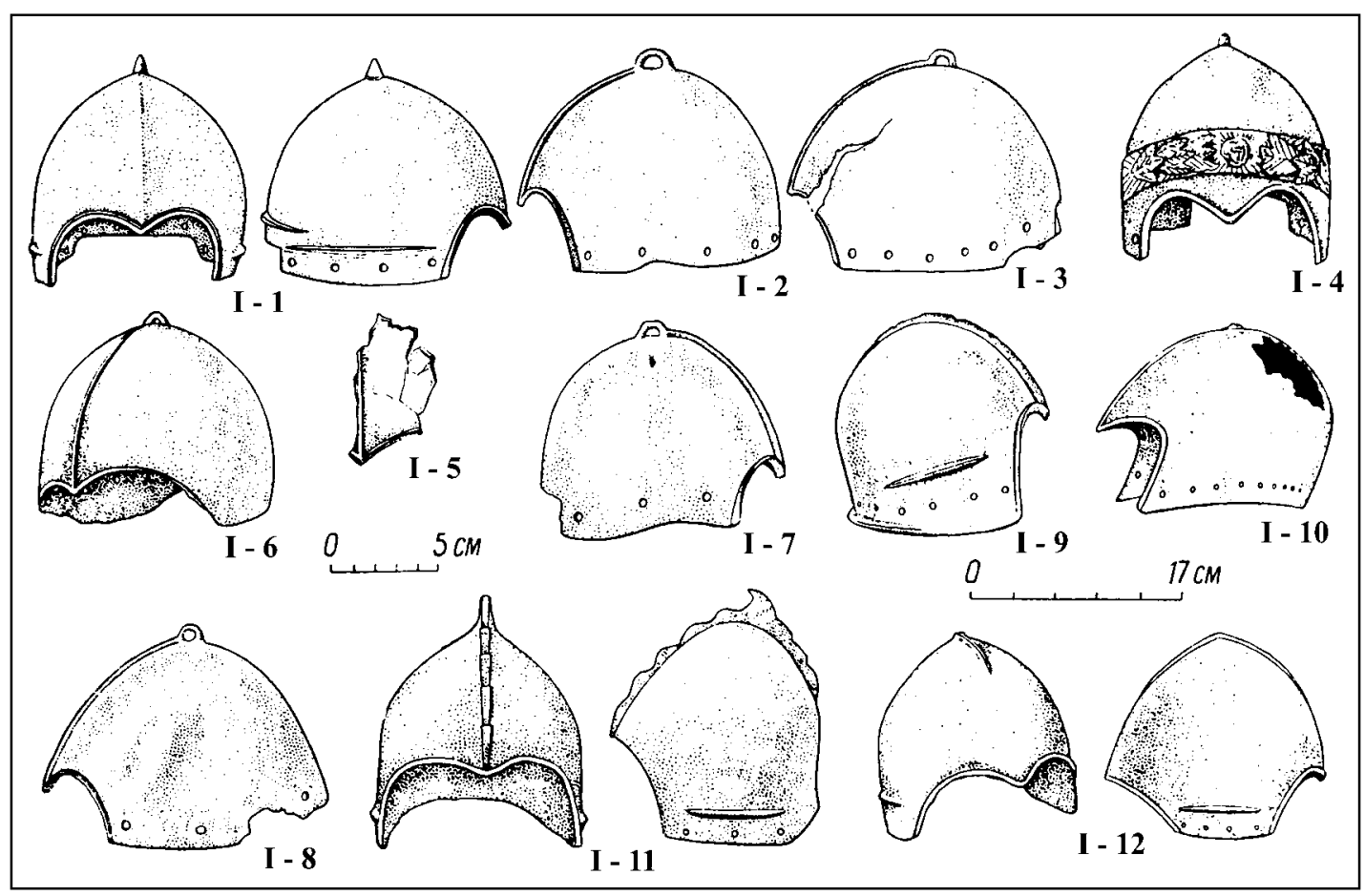

Fig. I. Cascos "Kuban”, según Galanina, 1985, Fig. 1. 1-2) Kelermes, kurgano 2 B. 3) Kelermes, kurgano 1 III. 4) Krymskaja, kurgano Voroncovskij. 5) Krymskaja, kurgano Macepinskij. 6) Gvardejskoe. 7) Stary Pečeur. 8-9) Lugar desconocido. 10) Siberia occidental. 11) Samarkanda. 12) Kysmyci.

Salvo el primer casco de Krymskaja, se trata de cascos-capucha sin adornos que sobresalían por delante sobre la frente, protegían la nariz con un pico, tapaban las orejas por los lados y por detrás, y se prologaban hasta la nuca. Se ponían igualmente sobre capuchas de cuero y parecen estar copiados de modelos de cuero, en caso de que el saliente prominente -en general desde la nariz hasta la coronilla - represente el vestigio de una costura. En el casco de Samarkanda esto se ha convertido en una "cresta" cuya forma es característica de las representaciones de grifos de Pazyryk. La denominación de este tipo de cascos se escogió de acuerdo con la concentración de hallazgos en la región de Kuban, que para muchos autores indica también un origen norcaucásico de los cascos, mientras que Galanina los relaciona con modelos de Asia Occidental. Por el contrario, Kuz'mina, Kopylevy otros excavadores se han pronunciado a favor de un origen centroasiático de esta forma de casco, aduciendo, entre otras, la presencia de molibdeno en el bronce del casco de Kysmyci de la región de Dzambul. A este respecto debería tenerse en cuenta la presencia de cascos-capucha de bronce de esta forma en las tumbas de Anyang de la época Shang de China ${ }^{19}$ (Fig. II) así como la coincidencia de estos cascos en la protección del cuello.

De la época Chou (a partir del siglo XI a. de C.) proceden asimismo cascos de este tipo que se asemejan aún más a los cascos "Kuban". Según Gorelik ${ }^{20}$, en las tumbas había cuatro cascos junto con bronces de Karasuk. Otro casco de Sin'iun se encuentra en el museo de Toronto. Un casco con yelmo adornado con calados(Fig. III, 2) se guardaba en Bejfu. Llama

19 Müller-Karpe, 1980, Taf. 583, B, C, D und Taf. 587,1-2.

20 Gorelik, 1987, Abb. 6 u.a. 
la atención la anilla fundida dispuesta en la "punta" de varios cascos, en la que probablemente se sujetaba un adorno.

Los hallazgos de cascos "Kuban" guardan relación con los de tipo escita -Kelermes-, sármata — Stary Peceur-, saca — Kysmyci—y sogdiano (?) —Samarkanda— quizás atribuible a un mercenario saca. Se delimitó la datación por el hallazgo del kurgano de Kelermes y de los cascos del lago Cetuk, es decir, dichos cascos estuvieron en uso desde el siglo VI hasta el siglo I a. de C.

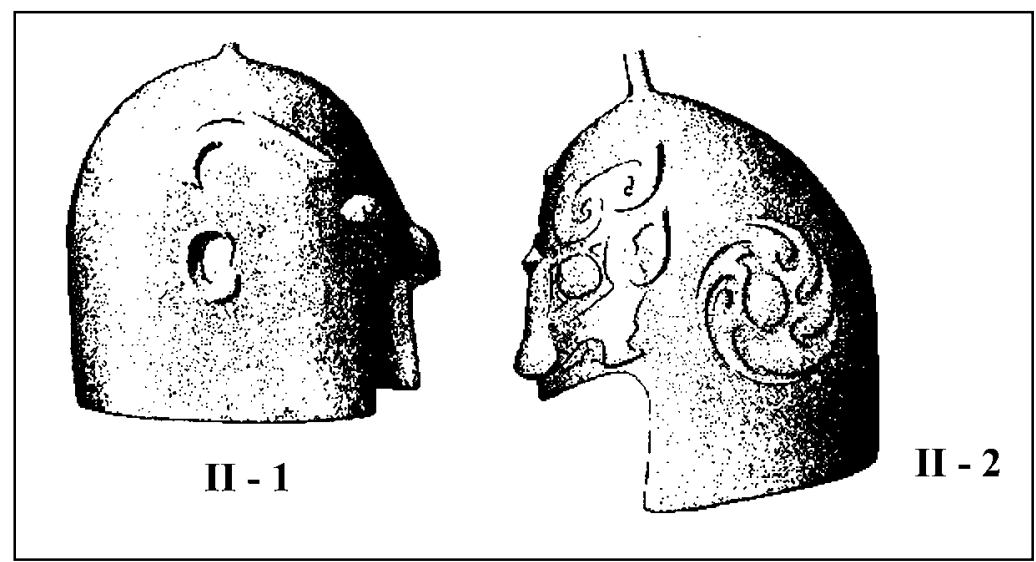

Fig. II. 1-2) Cascos-capucha de la época Shang, Anyang. Según MüllerKarpe, 1980, tabla 587, 1, 2.

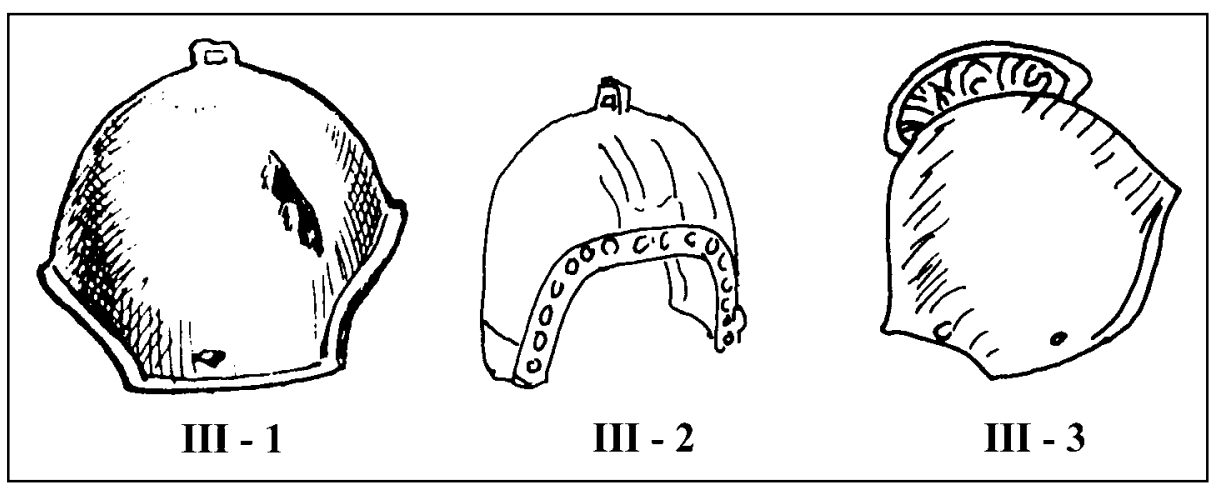

Fig. III. Cascos del tipo llamado "Kuban" de Asia. Según Gorelik, 1987, Figs. 6, 11, 12, 13. 1) Casco del Museo de Toronto, siglo VIII-VII a. de C.. 2) Casco de Bajfu en Pekín, siglo IX-VIII a. de C.. 3) Casco de Nan'sangen', Ljponin, siglo VII a. de C.

Un segundo grupo de cascos utilizados por los escitas lo formaban las importaciones griegas con máscaras para la cara, o con protectores para la nariz como en el yelmo de Solocha, procedentes del kurgan I de Kelermes, el kurgan Kekuvatskogo, de Pantikapeion, Solocha y Gorgippija ${ }^{21}$, Romejskovka ${ }^{22}$, Pastyrskoe, el bosque de Galušino ${ }^{23}$ (Fig. IV) y el kurgan

21 Košelenko, Kruglikova, Dolgorukov, 1984, Taf. LXXXI, 13, 30, 35, 40. 
de la Staniza Kurdzipskaja ${ }^{24}$. Hasta ahora ha quedado como pieza aparte un casco de bronce de forma parecida a la asiria de Klin-Jar en Kislovodsk. (Fig. VI, 5). Procede de una tumba de la cultura Koban y parece ser la copia de un casco asirio puntiagudo ya que está doblado y remachado, y no fundido ${ }^{25}$.

En los siglos II y I a. de C. llegaron nuevas formas de cascos a las estepas y a Crimea. Así, un casco del grupo "tracio" con orejeras, del siglo III-II a. de C., procede de Neapolis en Crimea $^{26}$, un casco parecido a los modernos cascos de bombero, del siglo IV-II a. de C., procede de Kertsch ${ }^{27}$ y una caperuza puntiaguda con carrilleras, del siglo Ia. de C., procede de la Staniza Achtamsovka ${ }^{28}$ (Fig. V). El casco de Neapolis puede relacionarse con el de Bubuja (Fig. VII), o incluso es posible que ambos sean idénticos y solamente se diferencien en el dibujo. De las tumbas sármatas de Novoprochorokova y de Veselej29 tenemos cascos de bronce en forma de gorras de jockey tales como las que aún llevan los jinetes en la actualidad (Fig. V, 2-3). Se parecen a los cascos celtas de Giuboasco o de Amfreville ${ }^{30}$.Esta forma de casco aparece todavía en la época post-sármata como puede observarse en los hallazgos de las tumbas en Nikol'skaja ${ }^{31}$. Podrían guardar relación con la presencia de mercenarios gálatas-celtas en el Estado del Ponto.

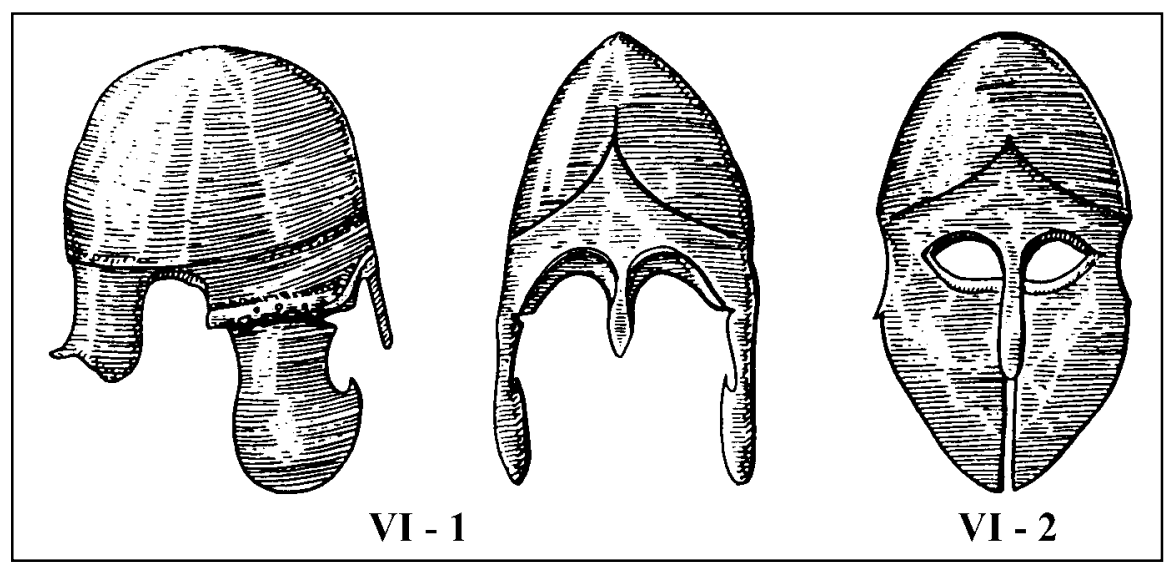

Fig. IV. Cascos de importación griega. Según Meljukova, 1989, Tabla 33, 22, 23. 1) Casco de un kurgan en Pastyrskoe. 2) Casco de un kurgan en Romejkova.

Smirnov ${ }^{32}$, que atribuye a los sármatas seis cascos de este tipo procedentes de Mar'ovka, Novoprochorovka, Antipovka, Veselej, Bol'soj Karaman y la Staniza Bergeevskaja, en Krasnodar, supone que siguen modelos italianos-celtas. Pero a pesar de toda esta semejanza,

\footnotetext{
22 Meljukova, 1989, Taf. 22, 23, 33.

Meljukova, 1989, Taf. 33, 22.

Galanina, 1980, Katalognr. 68-68.

Belinskij, 1990, S. 190-195.

Meljukóva, 1989, Taf. 54, 9.

Košelenko, Kruglikova, Dolgorukov, 1984, Taf. LXXXI, 41

Košelenko, Kruglikova, Dolgorukov, 1984, Taf. LXXXI, 46.

Meljukova, 1989, Taf. 65, 47 und 48.

Schlette, 1980, S. 36, Taf. 27.

Meljukova, 1989, Taf. 99, 32.

Smirnov, 1984, S. 71.
} 
parece poco probable una derivación la Galia hacia el Este, por ejemplo en la datación del casco de Mar'ovka en la época sármata antigua (siglo VI a V a. de C.), o bien la datación es errónea.

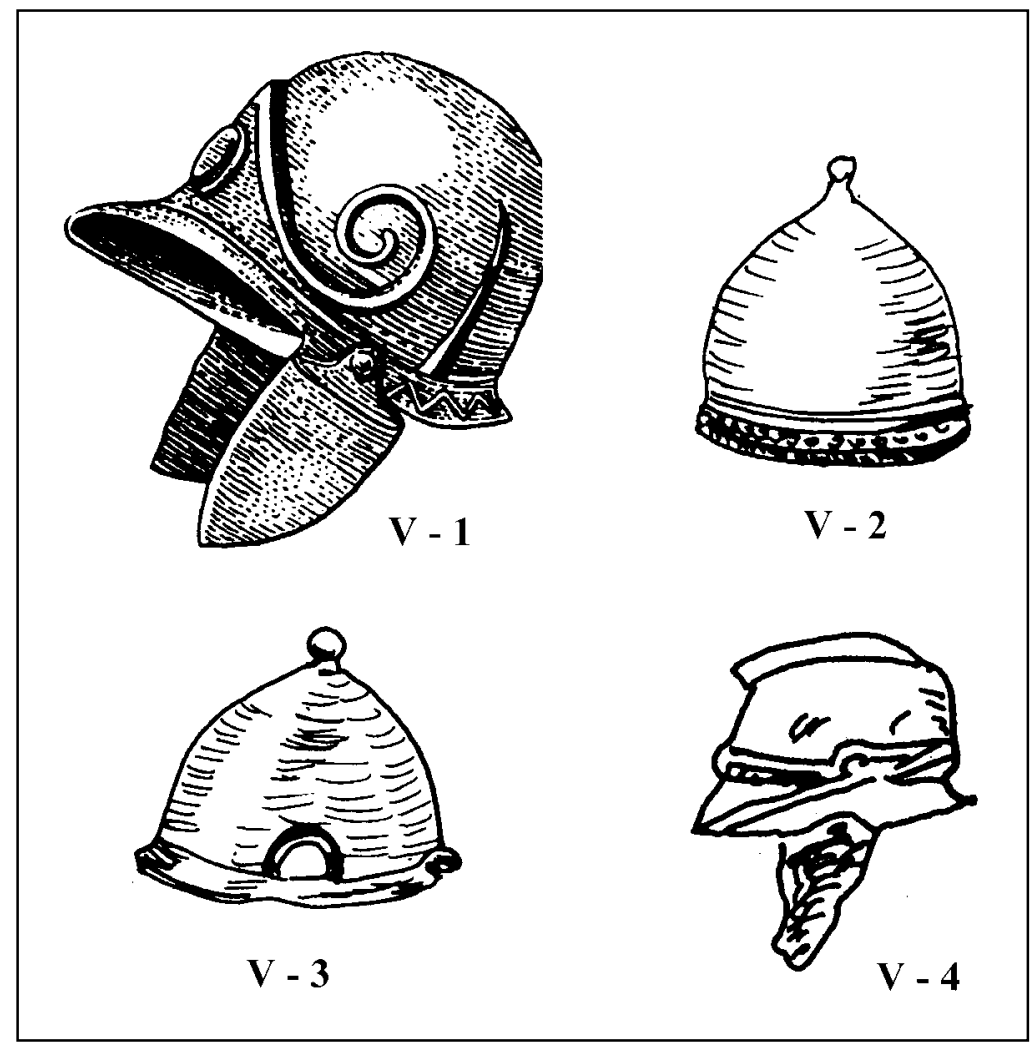

Fig. V. Cascos con paralelos romanos y celtas. 1-3 según Meljukova, 1989, Tabla 54,9; 65, 47, 48; según Košelenko, 1984, Tabla LXXXI. 41. 1) Casco de Neapolis, Crimea, crimeo-escita. 2-3) Cascos de Novoprochokova y de Veselej, sármatas. 4) Casco de Kertsch.

Un casco muy apuntado del campo de sepulturas sármata Grekii II al Noroeste del Cáucaso recuerda bastante las formas de los cascos asirios ${ }^{33}$.Salvo el casco de Neapolis no ha aparecido ninguno de estos cascos metálicos en combinación con placas protectoras, caso que se daba sin embargo en las capuchas guarnecidas con láminas protectoras, confeccionadas probablemente con cuero (Fig. VIII). Se trata de la tumba I del kurgan 6 en Aleksandrovka junto al Dnieper ${ }^{34}$, el kurgan de Novorosanovka junto al río Ingul en la región de Nikolsev y la tumba junto a la aldea de Gladkovščina ${ }^{35}$. Especialmente el "casco" del Ingul está bien conservado y ha sido excavado con exactitud de modo que el hallazgo es inequívoco. Una capucha con largas orejeras y protector para la nucaestá guarnecida con láminas de

\footnotetext{
33 Meljukova, 1989, Taf. 99, 32.

34 Kovaleva, Murchopad, 1982, S 91-102.

35 Šapošnikova, 1970, S. 208-2; Mhilulin, 1988, S. 116-126.
} 
protección $^{36}$ (Fig. VIII,1). En mejor estado aún se encontraban el casco, la armadura y el escudo de un guerrero del kurgan de Gladkovscina. El casco constaba de 775 láminas protectoras cosidas con el lado redondeado hacia arriba sobre una capucha de tela (Fig. VIII,2). Un casco parecido forma también parte de una estatuilla de Svestari en Bulgaria ${ }^{37}$. La estatuilla representa a un hombre con armadura (Fig. VIII,5). No se puede afirmar con seguridad que pertenezcan a esta clase los cascos de los jinetes con armadura y de los soldados de a pie reproducidos en los murales de la necrópolis de Pantikapeion ${ }^{38}$.

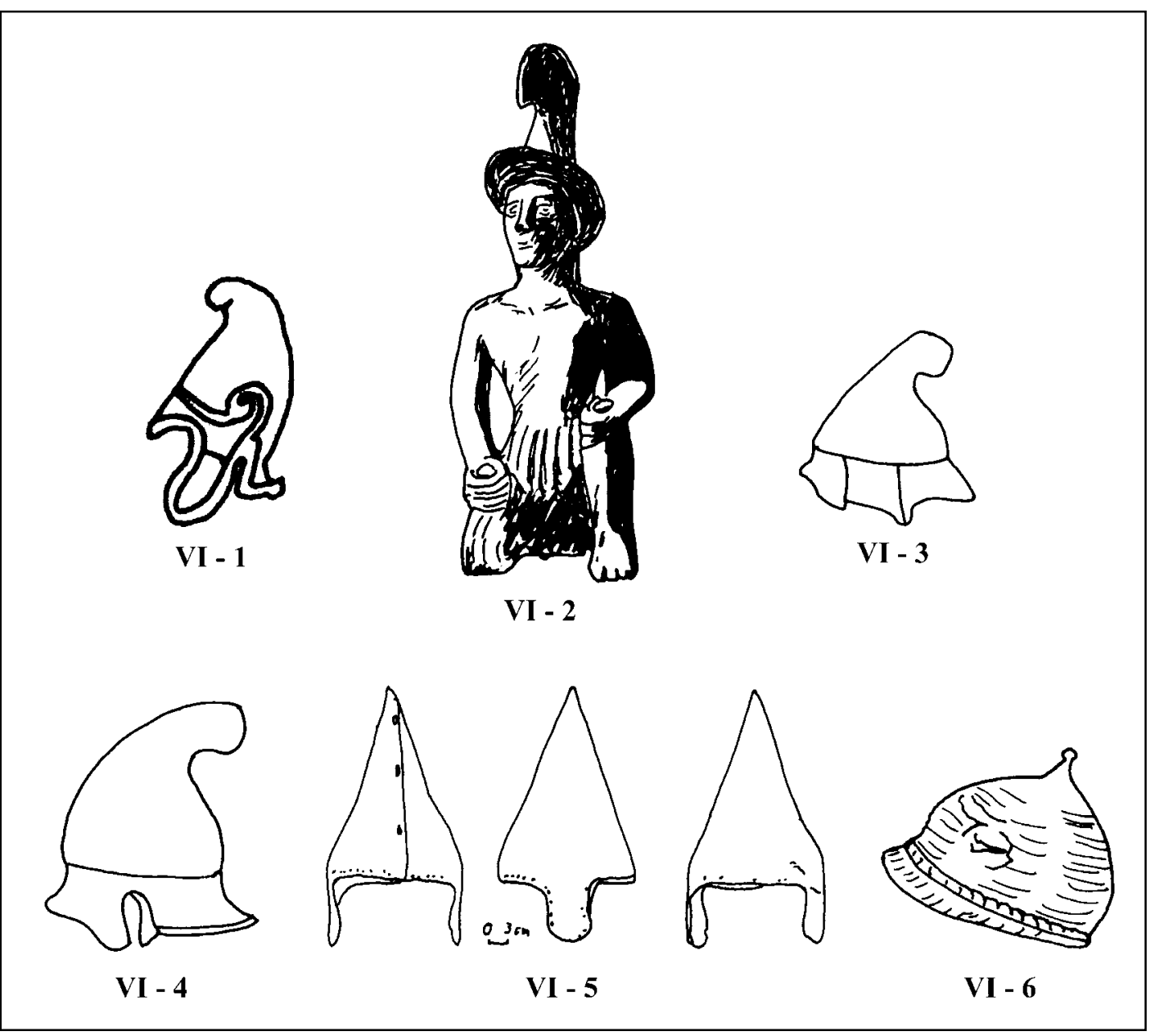

Fig. VI. Cascos en forma de gorro, 1 según Koselenko, 1984, Tabla LXXXI, 34; 2 según Debaine-Francfort, 1990, Fig. 1; 3-4 según Černenko, 1968, Fig. 45, 3, 4; 5 según Belinskij, 1990, Fig. 3; 6 según Meljukova, 1989, Tabla 81, 8. 1) Casco en forma de gorro "frigio", aquí escita, del kurgano Gran-Blisnitza. 2) Figura de guerrero con casco de "águila",cobre, Xinguan. 3-4) Cascos de Olonesty. 5) Casco asirio de bronce de Klin-Jar, siglo VII a. de C. 6) Casco-gorro post-sármatade Nikolskaja.

\footnotetext{
36 Šapošnikova, 1970, Abb. 4.

37 Berciu, 1974, Abb. 75.

38 Košelenko, Kruglikov Dolgorukov, 1984, Taf. XVII, 2, 3.
} 
Cernenko ha interpretado oportunamente las pinturas murales y las ha relacionado con fragmentos de cascos parecidos (ver nota 13). Sin embargo es posible que se trate de cascos de hebilla, que tendrían que haber aparecido por esta época en las estepas, ya que pueden verse en la columna de Trajano como parte de la armadura de los jinetes sármatas. H. von Gall pretende derivar el casco de hebilla de la corona iraní y señala a los sármatas como inventores de este casco $^{39}$. Sin embargo tendríamos que admitir entonces que este casco fue originalmente una versión de la capucha de cuero, que apenas puede aparecer en el material arqueológico. A este respecto, el casco procedente de la aldea de Jarysmardy en la región de los ingusch de Chechenia es el que se aproxima más a los cascos de la columna de Trajano ${ }^{40}$ (Fig. IX, 1). Sin embargo este casco data del siglo XII d. de C. y consiste en una capucha plana provista de multitud de remaches.

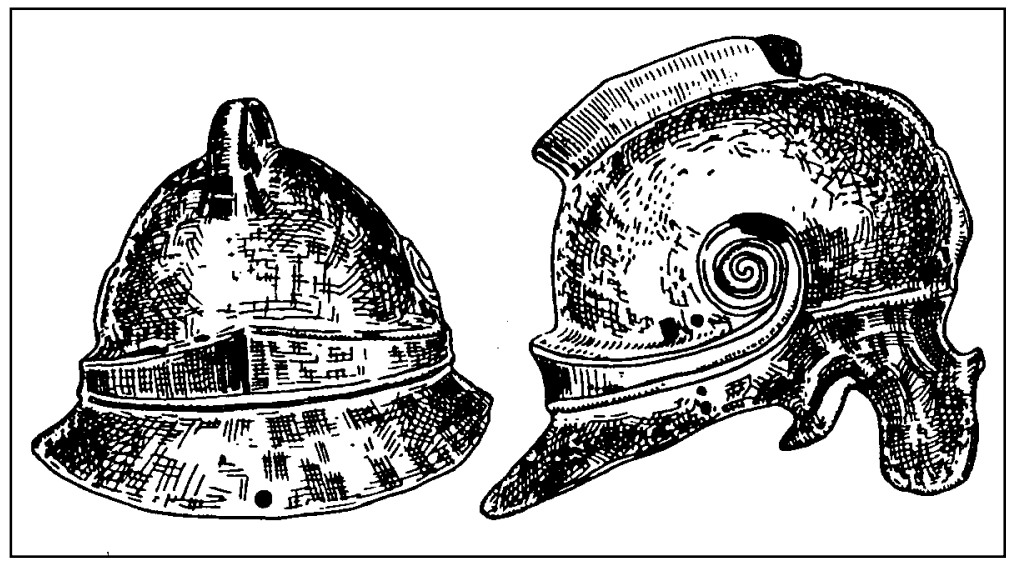

Fig. VII. Casco de Bubuja, Moldavia. Según Černenko, 1968, Fig. 7.

Habla en favor de la relación entre coronas y cascos de hebilla, admitida por H. von Gall un tocado femenino de oro y piedras preciosas encontrado junto con una máscara funeraria de oro $^{41}$ en Samsi en Kirguisia (Fig. IX, 2). Se ha fechado entre los siglos IV o V y muestra hebillas adornadas con piedras de colores entre láminas de oro. Resulta difícil de clasificar un adorno de oro de un tocado cónico de Ak-Burun ${ }^{42}$ (Fig. IX, 3), en el quetal vez se hayan reproducido en oro los bordados de los tocados "normales".

Los cascos de hebilla del tiempo de la invasión de los bárbaros ${ }^{43}$ — hacia el año 500 que se han encontrado en Europa Central y Occidental, y también en el Norte de África,son más altos y, en parte, primorosamente forjados. Como territorio de origen señalan al menos a Europa Oriental, pero también han aparecido ejemplares en Siberia Oriental, como en las excavaciones de Relka en el territorio del Obi-Irty ${ }^{44}$ (Fig.IX, 4). Más parecidos a los cascos de Europa Central y Occidental son los que se reproducen en las pinturas murales sogdianas de $\operatorname{Afrasiab}^{45}$ (Fig. IX, 7) y de Pandzikent ${ }^{46}$. Junto a ellos hay cascos planos, pintados de ne-

\footnotetext{
39 Gall, 1990; s. auch Ebert, 1929, S. 32-114, Taf. 48 C.

40 Mamaev, Cachkiev, 1982, S. 250-254.

41 Kanimetov, 1983, Nr. 140 und 141.

42 Artamanov, 1970, Taf. 272; Nerazik, Rapoport, 1978, S. 79-88, Abb. 4.

43 Werner, 1956; S. 32 und 78.

44 Kosarev, 1984, Taf. 22, 5.

45 Al'baum, 1975, Taf. LVII.
} 
gro, probablemente de cuero ${ }^{47}$. Posiblemente con este mismo material están confeccionados los cascos de las esculturas de estuco del palacio kuschan —o templo- de Khalcajan ${ }^{48} \mathrm{y}$ del castillo de Toprak Kala (Fig. XIII). Tienen el saliente central ancho sobre la coronilla igual que los cascos del grupo "Kuban". Este saliente central es la costura que se forma al unir dos semicasquetes. La identificación en cada uno de los cascos resulta problemática ya que el tocado de cabeza "normal" de los habitantes de las estepas, como se muestrapor ejemplo en las antiguas figuras de piedra turcas de Tuva ${ }^{49}$, tenía la misma forma. En ellas se muestran las orejeras protectoras del frío como precursoras de las carrilleras (Fig. IX, 6). Es muy posible que este tipo de gorros estén representados en una parte de las esculturas de la época Kuschan.

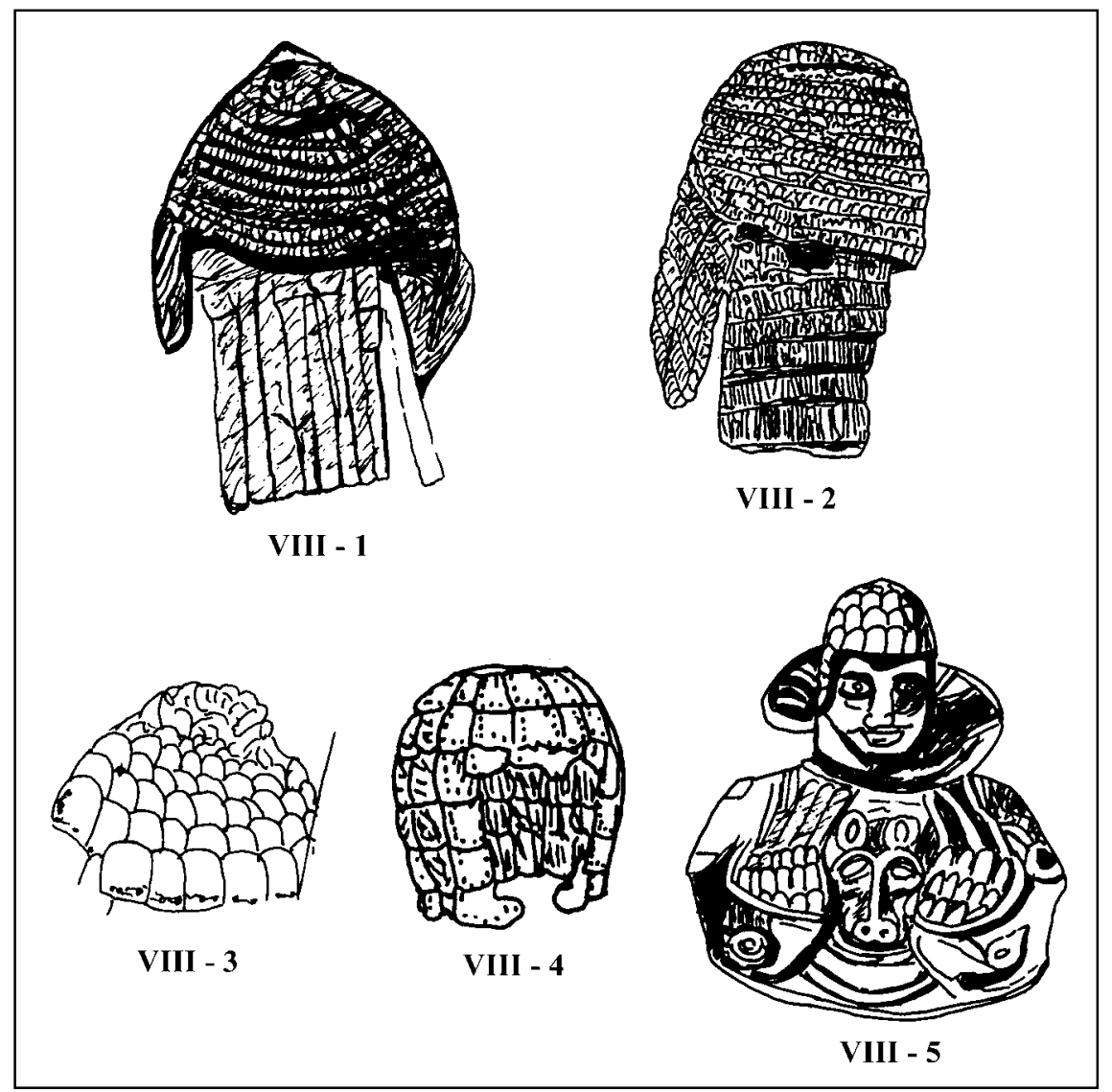

Fig. VIII. Gorros-casco. 1 según Šapošnikova, 1976, Fig. 4; 2-3 según Minzulin, 1988, fig. 1, 5; 4 según Gorelik, 1987, Tabla 6, 10; 5 según Berciu 1974, Fig. 75. 1) Casco-gorro reforzado del kurgano de Novorosanovka. 2-3) El casco de Gladkovščina, reconstrucción, hacia el año 400 a. de C. 4) Casco chino reforzado con placas, tumba 44 en Ujanstaj, Hebei, siglo III a. de C. 5) Hombre con armadura y gorrocasco, estatuilla de Svestari, Bulgaria.

\footnotetext{
46 Belenickij, 1973, S. 19, Taf. 28, 29.

47 Belenickij, 1973, Taf. 24, 32 u.a.

48 Pugačenkova, 1966, Taf. XXVI und XXVII; Pugačenkova, 1979, Taf. 116 und 119.

49 Evtjuchova, 1952, Abb. 57, 2.
} 


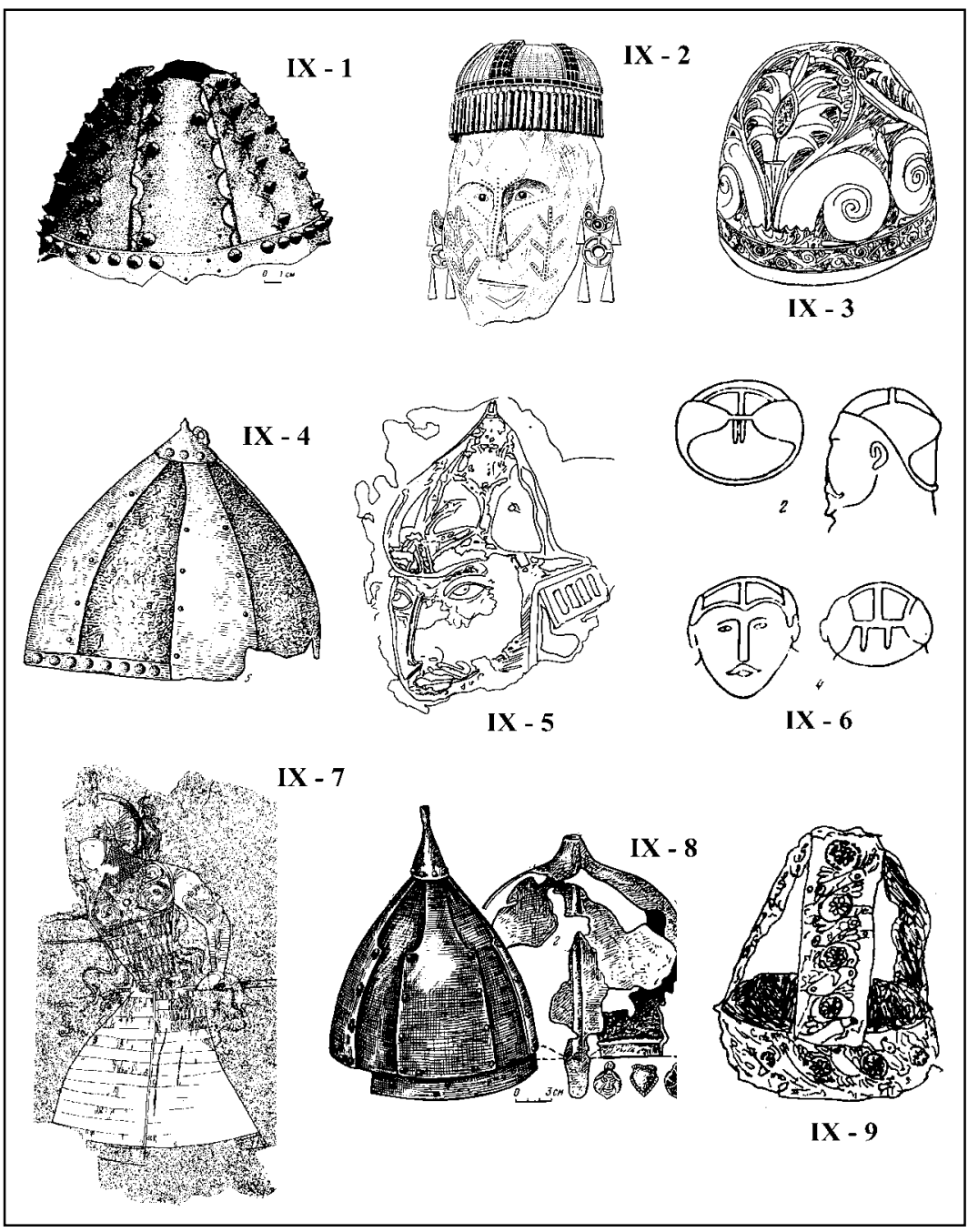

Fig. IX. Cascos de hebilla. 1 según Mamaev, Cachkiev, 1982, Fig. 1; 2 según Pletneva, 1981, Fig. 10,16; 3 según Artamanov, 1970, Tabla 272; 4 según Kosarev, 1984, Fig, 22,5; 5 según Al’Baum, 1975, Tabla LVII: 6 según Evtuchova, 1952, Fig. 37,2; 7 según Belenizki, 1980, p. 109; 8 según Pletneva, 1981, Fig. 55, 2, 2; 9 según Ebert, 1929, Tabla 38 C. 1) Casco de hebilla plano de la aldea de Jarysmardy, Cáucaso septentrional. 2) Capucha de hebilla de oro con máscara mortuoria de oro, Samsi, Kirguisia, siglo IVV. 3) Adorno de oro de una capucha cónica, Ak-Burun. 4) Casco de hebilla del campo de sepulturas de Belka, región del Obi-Irty, siglo V-VII. 5) Casco de hebilla, fragmento de las pinturas murales de Balalyk-Tepe, siglo VI-VII, copia. 6) Tocado de cabeza de una estatua de Tuva, $\mathrm{N}^{\circ}$ 37. 7) Casco de hebilla con protección de cota de malla sobre armadura de placas. Pintura mural de Pandzikent, espacio VI, 53. 8) Casco de hebilla con casco-gorro, derivado del casco de hebilla, cultura Karajukubov. 9) Partes de oro del casco-corona de Ositnjazka, comarca de Cirigin, siglo VIV a. de C. Se ha interpretado también como guarnición de un recipiente de madera. 
Por el contrario es seguro que, en las estatuillas chinas de los siglos IV-III a. de C. ${ }^{50} \mathrm{o}$ en la figura ecuestre de un "altar de fuego" del valle del Talas ${ }^{51}$ (Fig. X, 1), se trata de cascos que llevan igualmente un saliente central y carrilleras. Al menos un casco de esta clase se presenta en la tumba del príncipe del reino $\mathrm{Cu}$ en Lejgudun, provincia de Hubei. Dicho casco se enterró con el difunto en el año 433 a. de C..$^{52}$. Lleva saliente central y protecciones laterales. Otra escultura de Chalcajan ${ }^{53}$ ofrece una forma más del casco de cuero (Fig. X, 2). Parece remontarse al "baschlyk" (especie de capucha larga) de los nómadas.

Los cascos que recuerdan a los cascos-capucha chinos pueden verse en la "placa de guerra" del kurgan de Tepe ${ }^{54}$. Allí se encuentran capuchas-casco que llegan hasta muy abajo de la nuca y llevan largas "colas" de pelo sujetas en la coronilla, como las que colgaban probablemente de las anillas de los cascos "Kuban" (Fig. XVII). A la vista de la ausencia casi total de cascos originales al Este del Ural — con excepción de los ejemplos mencionados anteriormente- podría concluirse que aquí predominaban por lo general las capuchas de cuero, si no fuera por que las pinturas murales y los cuencos de plata de Sogdiana y de Bactriana demuestran la existencia de varias formas de cascos con hebilla, cascos-capucha, cascos puntiagudos ${ }^{55}$ y cascos de tres puntas ${ }^{56}$.

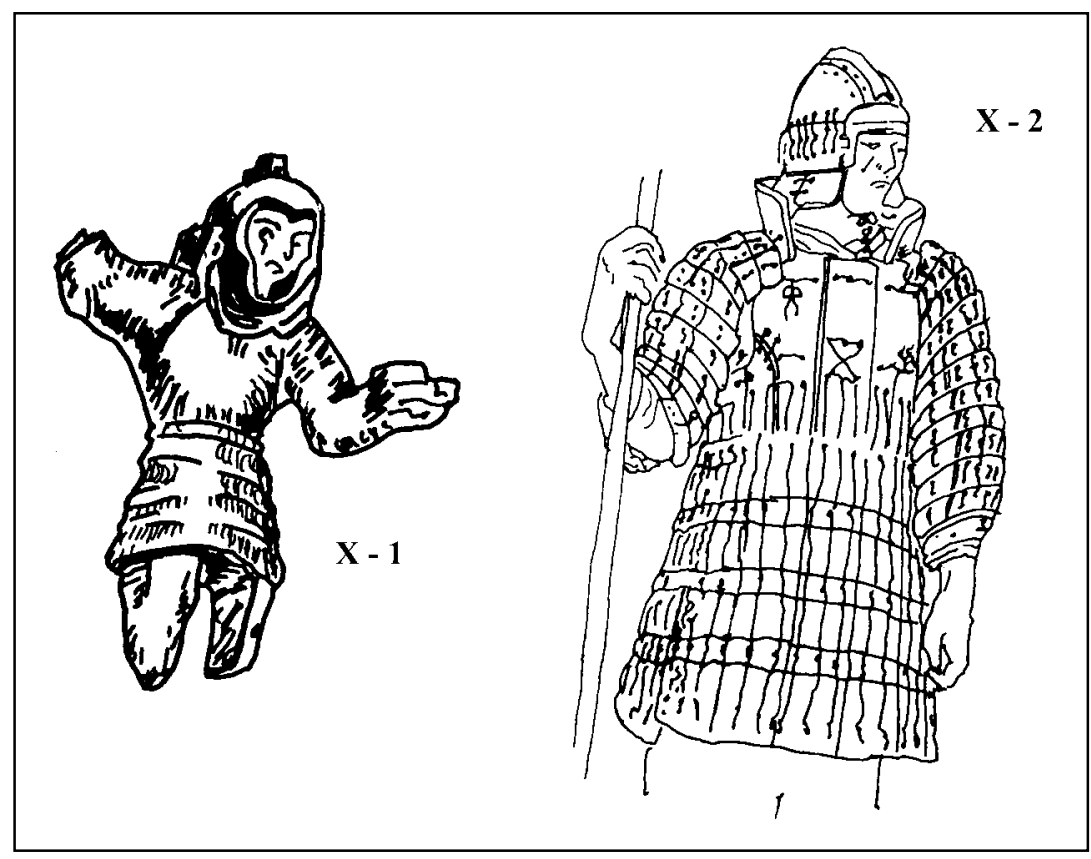

Fig. X. Cascos y armaduras, 1. Senigova, 1968, Fig. 1, 1, 5; 2 según Gorelik, 1987, Fig. 4, 1. 1) Jinetes con armadura de un "altar de fuego" saca, valle del Talas, Kirguisia. 2) Casco y armadura de la tumba de Lejgutun, Hubei, 433 a. de C.

50 Gorelik, 1987, Abb. 3, 6.

51 Gorelik, 1987, Abb.2, 2.

52 Gorelik, 1987, Ab b. 4, 1.

53 Pugačenkova, 1966, Taf. XIX.

54 Pugačenkova, 1987, S. 57.

55 Belenizki, 1980, S. 109, 129, 116, Taf. 51-54 u.a.

56 Belenizki, 1980, S. 49, Taf. 13. 
Los cascos de tres puntas aparecen, como parte de la armadura (Fig. XII), en la escena de dos combatientes del cuenco de Kulagys. En un mural del segundo templo de Pandzikent (Fig. XIV), los jinetes que se dirigen al templo llevan en la cabeza tocados parecidos. En este caso se podría tratar de coronas ya que el tema se remonta al cuadro budista de la comitiva de creyentes para la distribución de las reliquias de Buda.

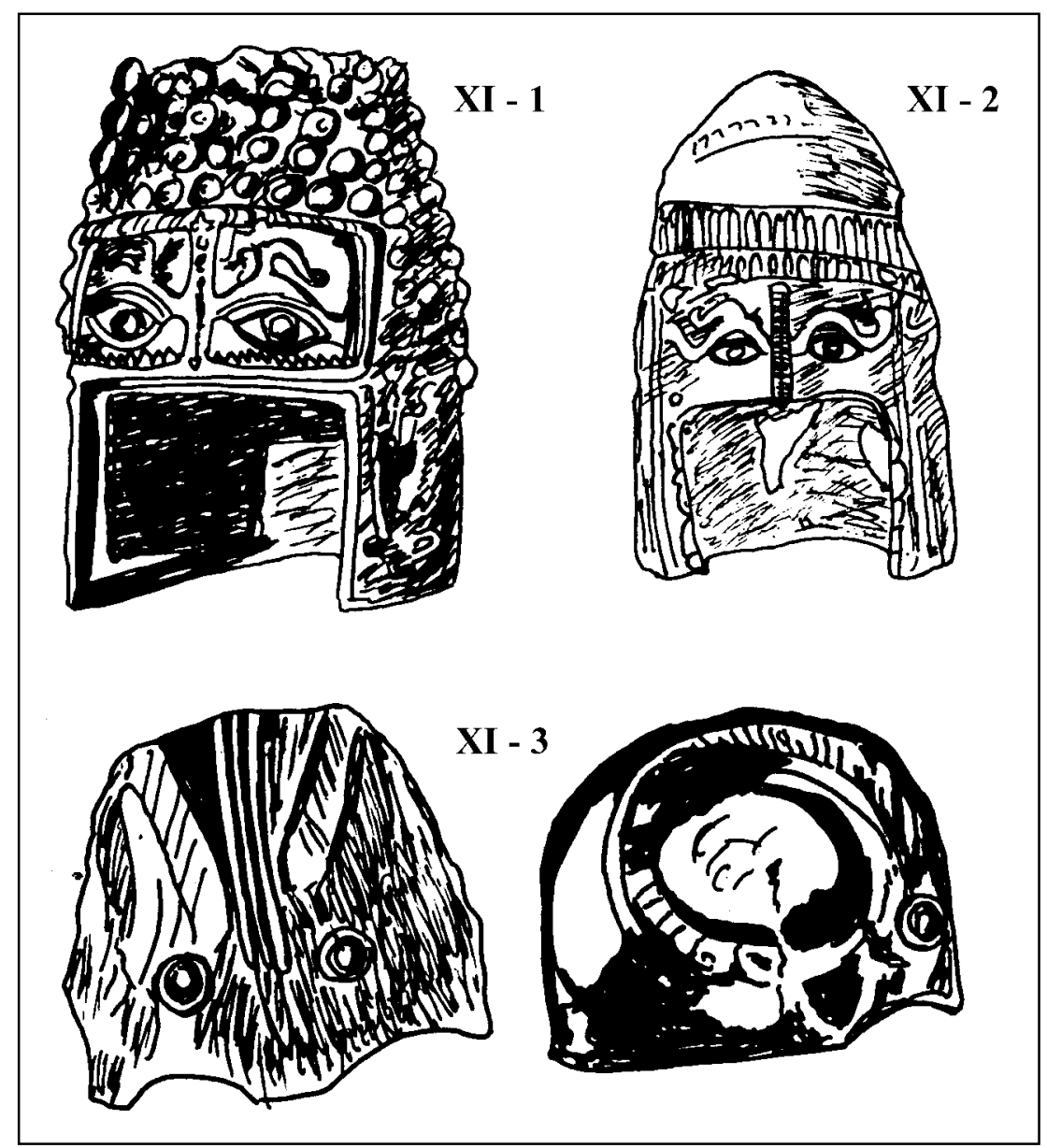

Fig. XI. Cascos de metales preciosos, 1 según Berciu, 1974, Fig. 40; 2 según Berciu, 1974, Fig. 5; 3 según Cernenko, 1968, Fig. 53. 1) El casco de oro de Cotofenesti, Tracia. 2) El casco de plata de Agighiol, Tracia. 3) El casco de la Staniza Dachovskaja.

En un antiguo dibujo turco por incisión de Kudyrge en el Altai ${ }^{57}$ se reproduce una corona de tres puntas (?). Parece tratarse igualmente de una "corona", extendida por los territorios boscosos de Europa oriental-Siberia, perteneciente a guerreros o a demonios de la guerra, que ha sido representada repetidas veces hasta la alta Edad Media ${ }^{58}$. Los dibujos por incisión

57 Pletneva, 1981, Abb. 21, 9.

58 s.u.a. Kosarev, 1991, Taf. 73, 1 und 74, 24. 
sobre bronce del territorio fluvial del $\mathrm{Obi}^{59}$ reproducen figuras portadoras de los correspondientes gorros de tres puntas junto a portadores de "cascos" con cabezas de animales y otros tocados. Algunas de las figuras de portadores de gorros con puntas llevan dos espadas en las manos, o son - ya en las placas de principios de la Edad del Hierro- rostros con una verdadera corona dispuestos entre dos puñales ${ }^{60}$. La representación más antigua del "gorro de tres puntas" - conocida por el autor - se puede ver en una placa de la cultura Tasmin del territorio fluvial de Cernovaja en la región de Minussinsk. La placa se puede fechar en los principios del tercer milenio a. de $\mathrm{C}^{61}$. Un ser con tres ojos y rostro redondo lleva esta corona. A su lado se encuentran dos lanzas (Fig. XX). En este caso debe tratarse de un antiguo símbolo de seres guerreros y asimismo los "caballeros" del cuenco de Kulagys pertenecerían también a esta tradición.

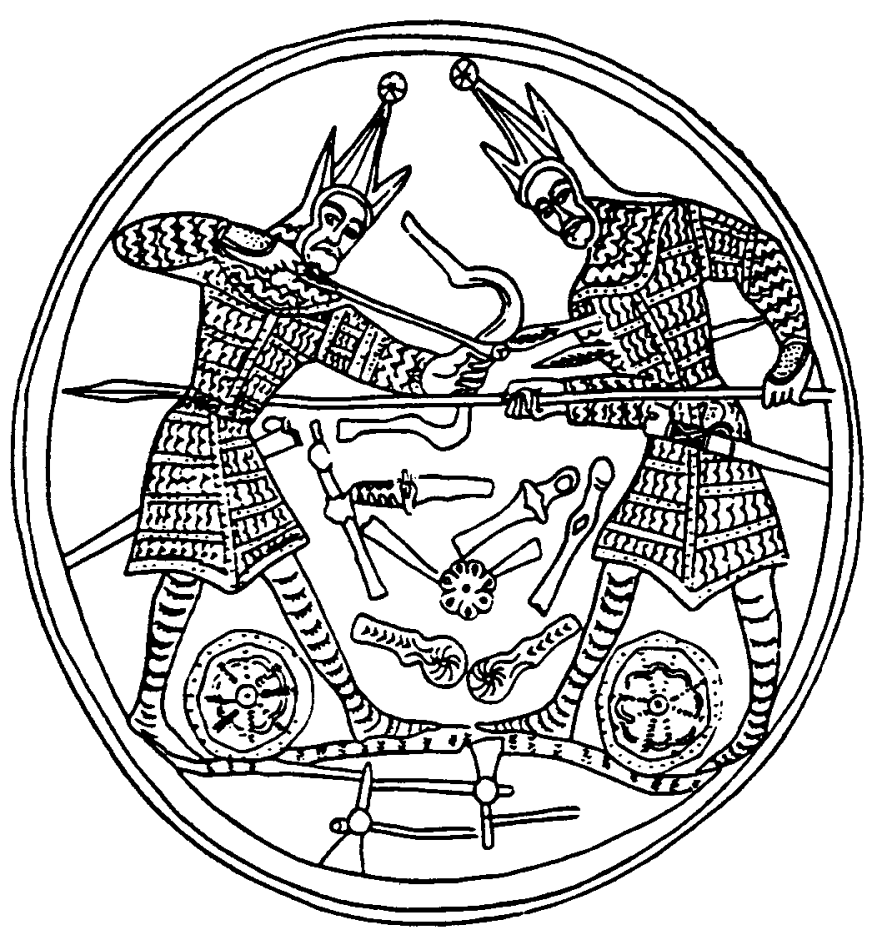

Fig. XII. Combate entre dos guerreros representado en el cuenco de plata de Kulagis. Según D’jakonov, 1954, Fig. 8.

Por el contrario, hasta ahora no se han podido hallar vestigios del casco de hebilla, a no ser por la "capucha" de Ak-Burun (ver Fig. IX, 3). Entre los cascos de hebilla de las escenas de combate de Pandzikent se encuentra una forma aparentemente derivada de las coronas sasánidas de la época tardía. El casco lleva sobre la frente la media luna envuelta por un círculo. Este mismo símbolo se dispone en el extremo de un largo vástago sobre la coronilla del hombre. Representa, tal vez, a un sasánida (Fig. XVI).

\footnotetext{
59 Černecov, 1953, Taf. XII, 1, 2, 4 u.a..

60 Kosarev, 1991, Abb. 74, 24 und 71, 17.

61 Vadeckaja u.A. 1980, Abb. 114.
} 


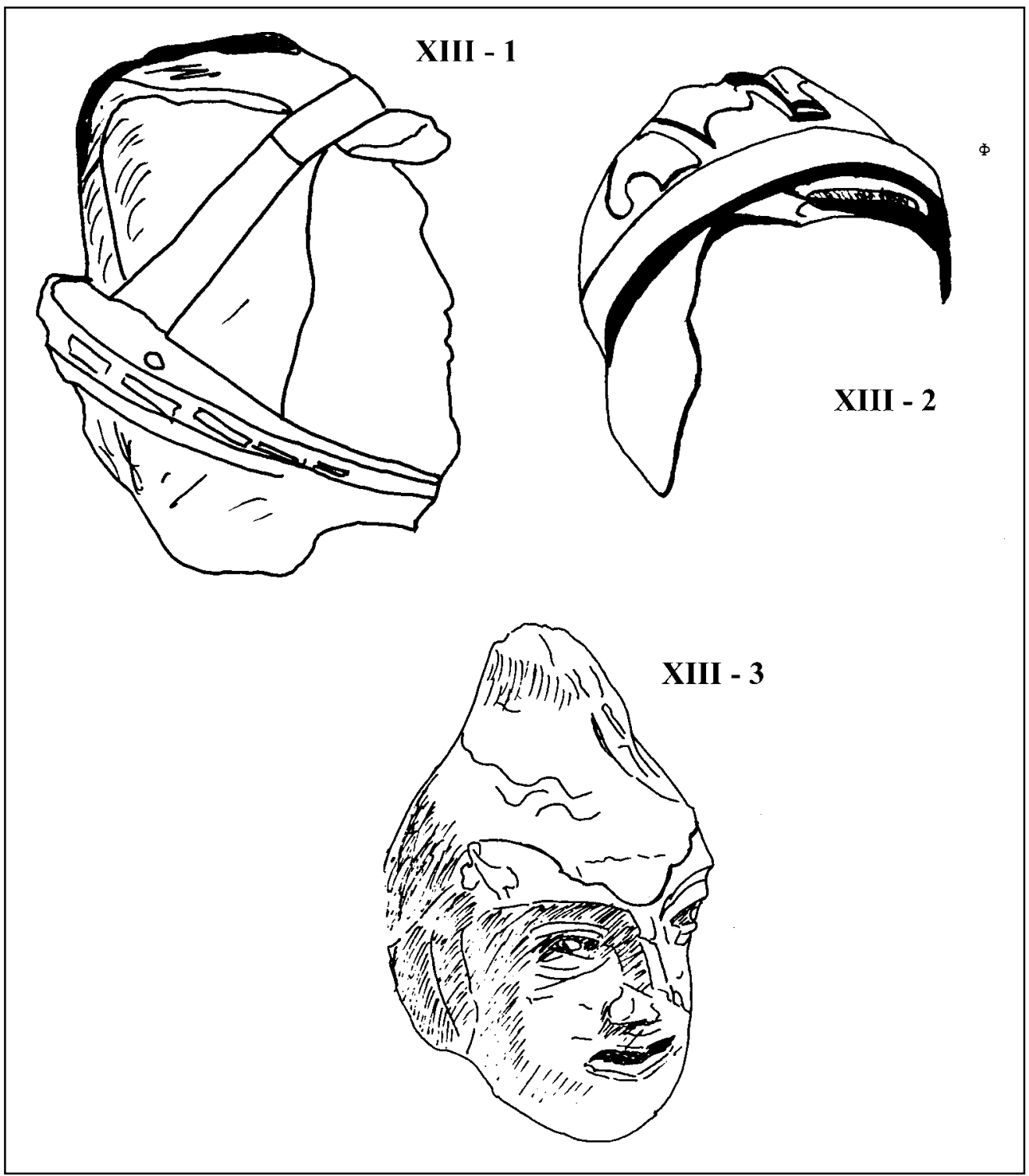

Fig. XIII. Cascos de cuero, 1-2 según Pugačenkova, 1979, Tabla 116; 3 según Pugačenkova, 1966, Tabla XIX. 1-2) Casco de cuero (?) con saliente central de una escultura de estuco de Chalčajan, siglo I-II. 3) Casco de cuero (?) en forma de "baschlyk" de una escultura de estuco de Chalčajan, siglo I-II.

Estos cascos de hebilla llevan generalmente una protección circular de cota de malla para el cuello que, en parte, se prolongaen la cota del cuerpo y, en parte, cuelga sobre la armadura que cubre los hombros y la parte superior del pecho y de la espalda ${ }^{62}$ (Fig. XVIII). Los cascos originales de este tipo se llevaron todavía en las estepas europeas en los siglos XI y XII tal como puede verse en los polowszianos y los kumanos y en los portadores de la cultura

62 vgl. Belenizki, 1980, S. 49 und 109. 
Karajakubov (Fig. X, 8). Junto a ellos se usaban cascos-capucha y cascos cónicos, o bien capuchas-casco cerradas, cuya decoración se derivaba de la de los cascos de hebilla. Hasta ahora sólo es posible seguir en parte la historia de los cascos de la región de las estepas. Junto a las importaciones antiguas desde el siglo $\mathrm{V}$ a. de $\mathrm{C}$., se percibe una influencia china desde principios del primer milenio a. de C. lo más tarde. No pueden determinarse los comienzos de este desarrollo, que podrían ser cientos, y aún miles de años más antiguos, como se puede intuir por la corona de la cultura Tasmin. La confección de gorros de cuero de tipo casco podría ser más antigua de lo que se ha podido comprobar hasta ahora.

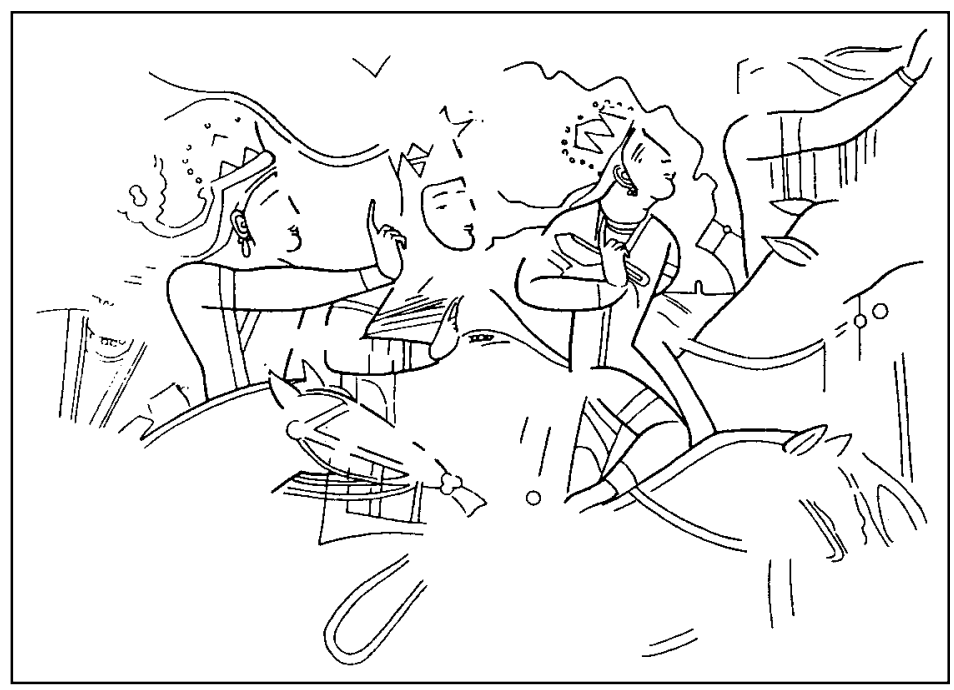

Fig. XIV. Coronas o "cascos con puntas" de un mural del segundo templo de Pandžikent, siglo VII-VIII. Según Belenizki, 1980, p. 49.

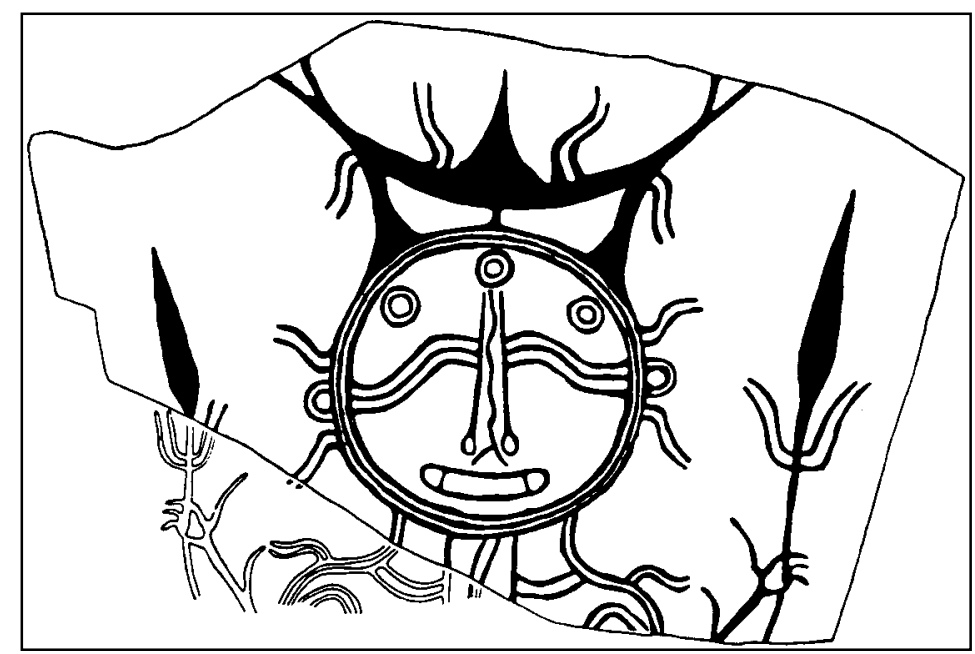

Fig. XV. Demonio de la guerra con corona de tres puntas, cultura Tasmin, región de Minussinsk, hacia 3000 a. de C. según Vadeckaja, 1980, Fig. 14. 
Tampoco está resuelta la procedencia y el primitivo desarrollo del casco de hebilla, mientras que la capucha reforzada con láminas protectoras parece ser obra de los pueblos de las estepas después de la adopción de la armadura con escamas, es decir, en el siglo VII o principios del VI a. de C.

N. del T. Algunos apellidos de autores así como algunas denominaciones geográficas presentan distinta ortografía a lo largo del texto. Por ejemplo "Belenizki"o "Belenizkij", "Kulagys" o "Kulagis", etc.

N. del A. Esto depende del original del que procede la referencia - ruso o alemán.

B. BRENTJES

Eberhardstr, 3 - D10367 Berlín (Alemania)

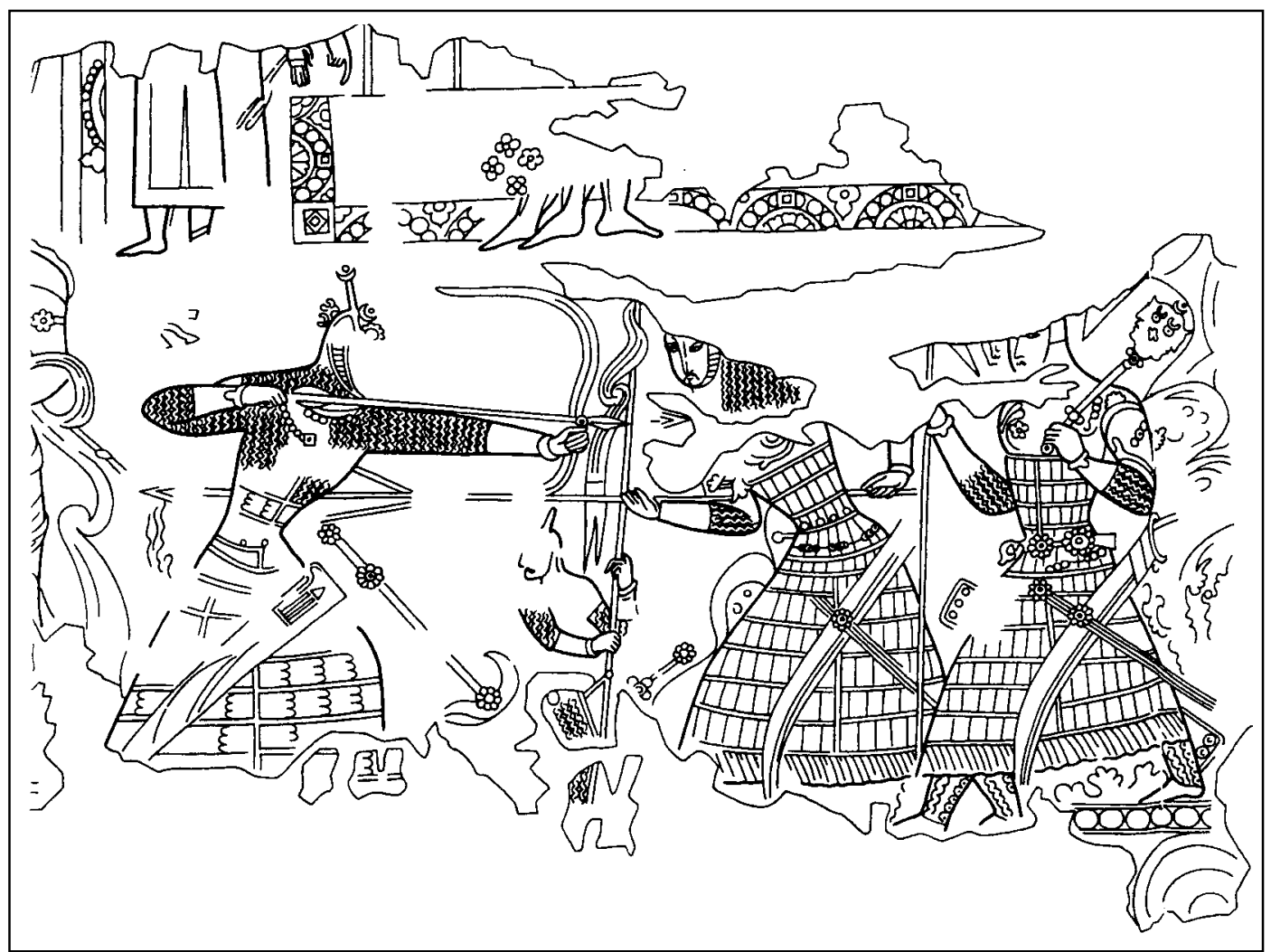

Fig. XVI. Casco-corona "sasánida" de una pintura mural de Pandžikent, siglo VII-VIII. Según Belenizki, 1980, p. 80. 


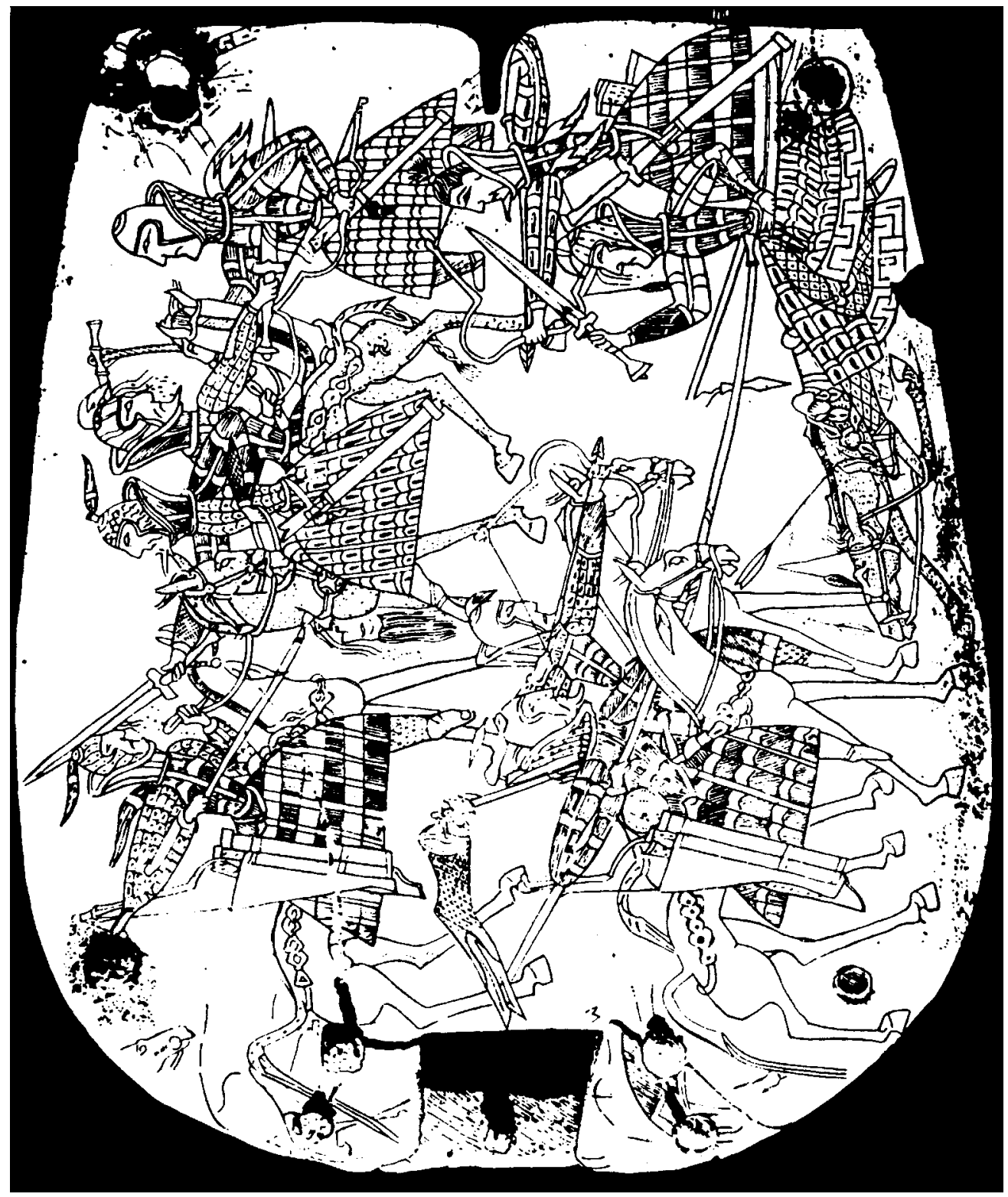




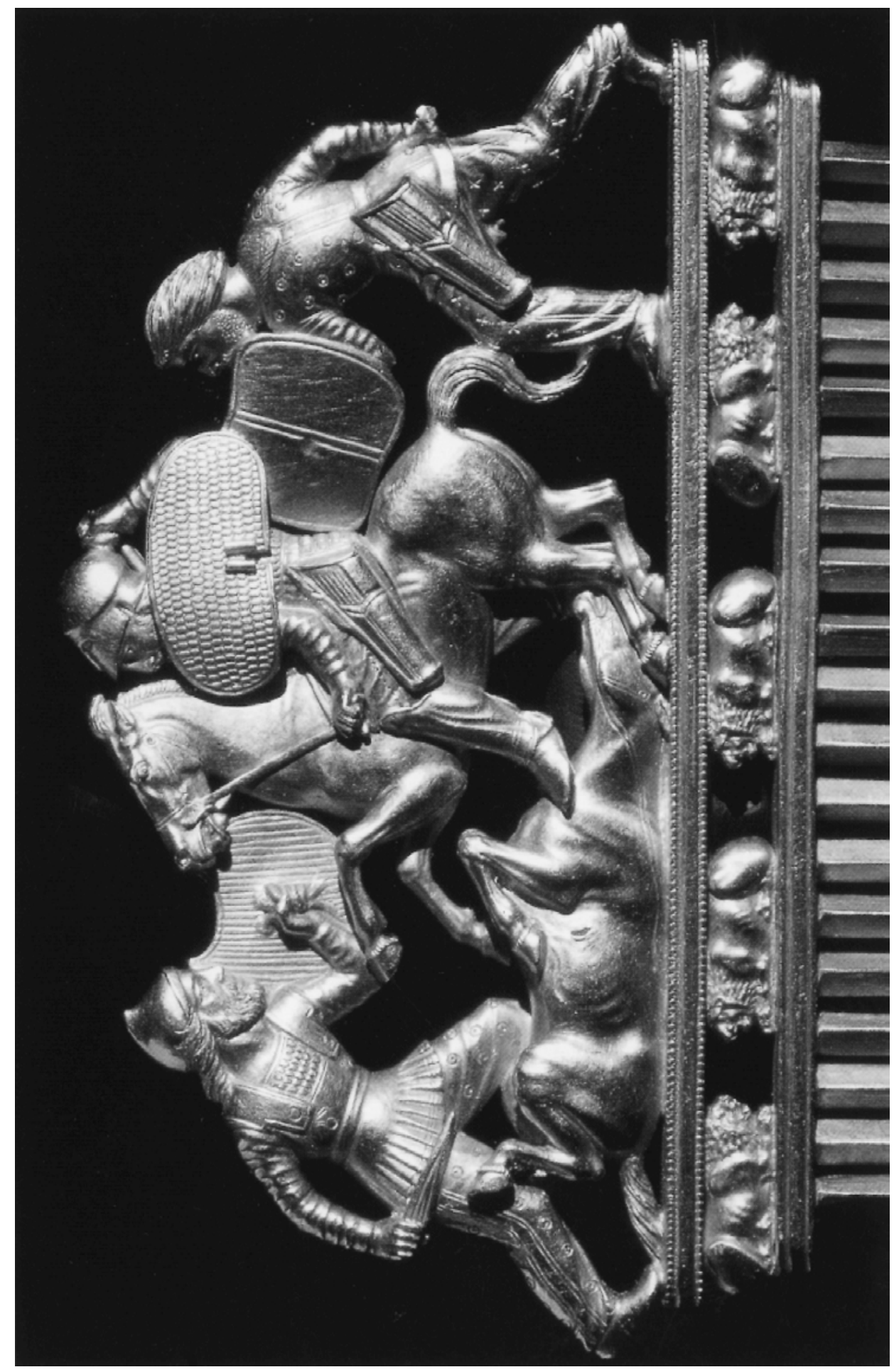

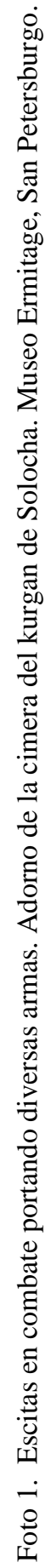



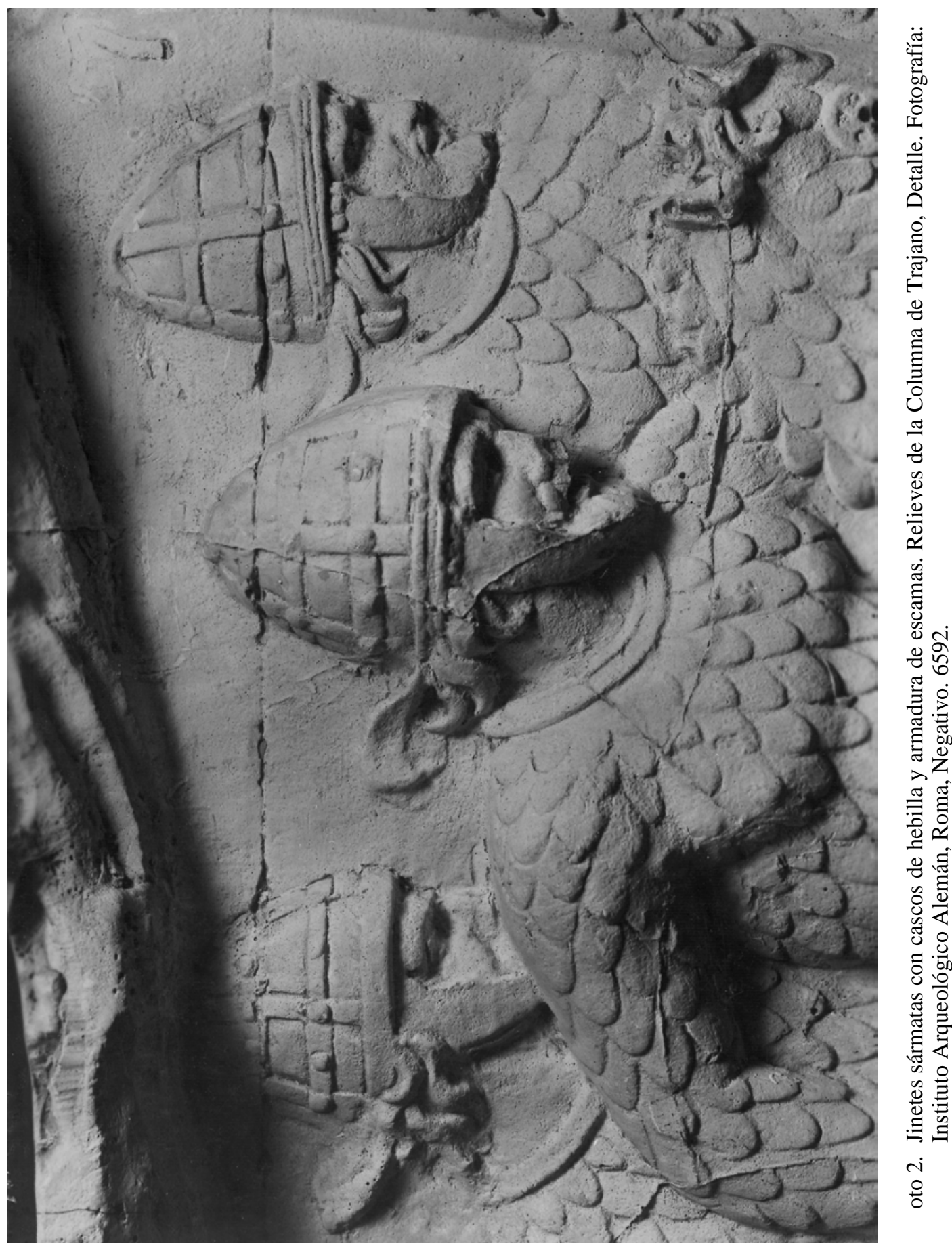


\section{BIBLIOGRAFÍA}

Abreviaturas:

AMI Archaeologische Mitteilungen aus Iran

IEJ Israel Exploration Journal

MIA Materialy i Issledovanija po Archeologii SSSR

SA Sovetskaja Archeologija

AL'BAUM, L.I. (1975): Živopis' Afrasiaba. Taschkent.

ARtamanov, M. (1970): Goldschatz der Skythen in der Eremitage. Prag- Leningrad.

BELENICKIJ, A.M. (1973): Monumental'noe Iskusstvo Pandžikenta. Moskau.

BeLENIZKI, A.M. (1980): Mittelasien. Kunst der Sogden. Leipzig.

BelinskiJ, A.B. (1990): K Voprosu o Vremeni Pojavlenija Šlemov Assirijskogo Tipa na Kavkaze. SA, 4, 190-195.

BERCIU, D. (1974): "Contribution à l'ètude de l'art Thraco-Gète". Bibliotheca Historica Romaniae XIII, Fig. 3-7. Bucarest.

BRENTJES, B. (1994): "Waffen der Steppenvölker I, Dolch und Schwert im Steppenraum vom 2. Jahrtausend v. Chr. bis in alttürkische Zeit". AMI, NF, XXVI, 5-45, Berlin.

BRENTJES, B. (1994): "Ortband, Rolltier und Vielfraß. Beobachtungen zur 'skythischen' Akinakes-Zier”, AMI, NF. XXVII, 147-164, Berlin.

BRENTJES, B. (1996): Arms of the Sakas (and other Tribes of the Central Asian steppes). Rishi Publications. Varanasi.

BRENTJES, B. (1997): "Waffen der Steppenvölker: Kompositbogen, Goryzt und Pfeil - ein Waffenkom-plex der Steppenvölker". AMI, XXVIII, 179-210, Berlin.

ČERNENKO, E.V. (1968): Skifskij Dospech. Kiew.

ČERnEzov, N.N. (1953): “Drevnjaja Istorija Nižnego Priob'ja”. MIA, 35, Moskau.

Debaine-Francfort, C. (1990): "Les Saka du Xinjiang avant les Han (206 av.-220 ap. J.-C.). Critères d'Idenfication". Nomades et Sédentiares en Asie Centrale, 82-95. Paris.

D’JAKonov, M.M. (1954): Rospisi Pjandžikenta i Zivopis' Srednej Azii. Moskau.

EBERT, M. (1929): "Südrußland, D Skytho-sarmatische Zeit". Reallexikon der Vorgeschichte, XIII, 32-114.

Evenari, M.; AHARoni, J. (1948): “The Ancient Desert Agriculture of the Negev. III Early Beginnings". IEJ, 8, 231-271.

EvTJuChOvA, L.A. (1952): “Kamennye Nazvanija južnoj Sibirii i Mongolii”. MIA, 24, Moskau.

GALANINA, L.K. (1980): Kurdžipskij Kurgan. Leningrad.

Galanina, L.K. (1985): "Šlemy Kubanskogo Tipa". En Bromlej, J.V. et al. (eds.), Kul'turnoe Nasledie Vostoka, 169-183. Moskau.

GaLL, H. von (1990): Das Reiterkampfbild in der iranischen und und iranisch beeinflußten Kunst parthischer und sasanidischer Zeit. Berlin.

GJERSTAD, E. (1935): "Die schwedischen Ausgrabungen auf Cypern”. Die Antike, 11. Berlin.

GoreliK, M.V. (1987): “Sakskij Dospech”. Central'naja Azija. Novye Pamjatniki Pis'menosti $i$ Iskusstva. Sbornik, 110-133. Moskau.

Kanimetov, A. et al. (1983): Pamjatniki Kul'tury i Iskusstva Kirgizii. Leningrad.

KoSAREv, M.F. (1984): Zapadnaja Sibir'v Drevnosti. Moskau.

Kosarev, M.F. (1991): Drevnjaja Istorija Zapadnoj Sibiri: Celovek i Prirodnaja Sreda. Moskau. 
KoŠElenko, G.A., KRUGLiKova, I.T. y V.S. Dolgorukov (1984): Antičnye Gosudarstva Severnogo Pričernomor'ja. En Archeologija SSSR. Moskau.

Kovaleva, I.F.; S.E. Muchopad (1982): "Skifskoe Pogrebenie Konca VI-V vv do n.E. u S. Alek- sandrovka". EnTerenožkin, A.I. et al. (eds.): Drevnosti Stepnoj Skifii, 91-102. Moskau.

LESKOv, A. (1990): Grabschätze der Adygen. München.

MAMAEv, Ch.M., D.J. ČACHKIEv (1982): “Šlem iz Selenija Jaryšmardy (Čečeno-Ingušetija)”. SA, 2, 250-254, Moskau.

MelJukova, A.I. (1989): "Stepi Evropejskoj Časti SSSR v Skifo-Sarmatskoe Vremja”. Archeologija SSSR, Moskau.

MiNŽULIN, A.I. (1988): “Zaščitnoe Vooruženie Voina-Lučnika V-IV v.v. do n.E. iz Kurgana Sel. Gladkovščina”. SA, 4, 116-126. Moskau.

MÜLLER-KARPE, H. (1980): Handbuch der Vorgeschichte, IV. München.

NERAZIK, E.E.; J.A. RAPOPORT (1978): “Die Festung Toprak-Kala in Choresmien”. Das Altertum, 24, 2, 79-88. Berlin.

Pletneva, S.A. (1981): “Stepi Evrazii v Epochu Srednevekov'ja”. Archeologija SSSR, Moskau.

PugaČEnKova, G.A. (1966): Chaľ̌ajan. Taschkent.

PugaČEnKova, G.A. (1979): Iskusstvo Baktrii. Epochy Kušan. Moskau.

PugAČEnKovA, G.A. (1987): “Obraz Kangjujza v Sogdijskom Iskusstve”. Iz chudožestvennoj Sokroviščnicy Srednogo Vostoka, 56-65. Taschkent.

ŠAPOŠNIKOVA, O.G (1980): “Pogrebenie skifskogo Vojna na R. Ingul”. SA, 3, 208- 212. Moskau.

SChlette, F. (1980): Kelten zwischen Alesia und Pergamon. Leipzig - Jena - Berlin.

SCHRÖDER, B. (1912): “Thrakische Helme”. Jahrbuch des Königlichen deutschen Archäologischen Institutes, XXVII, Berlin.

Senigova, T.N. (1968): “Osvetitel'nye Probory Taraza i ich Svaz's Kultom Ognja”. SA, 1, 208225. Moskau.

SMIRNOv, K.F. (1984): Sarmaty i Utverždenie ich Političeskogo Gospodstva v Skifii. Moskau.

VAdeCKAJA, E.B. et al. (1980): Pamjatniki Okunevskoj Kul'tury. Leningrad.

WERNER, J. (1956): "Beiträge zur Archäologie des Attila-Reiches". Bayerische Akademie der Wissenschaften. Philosophisch-Historische Klasse. Abhandlungen NF. H. 38 A. München. 